\title{
Base Station Antenna with Enhanced Cross Polarization Discrimination Performance by Using Horizontal Meandered Dipoles and Vertical Parasitic Elements
}

\author{
Orhan Murat Kadagan', Ceyhan Turkmen ${ }^{1}$, Mustafa Secmen ${ }^{\text {* }}$ \\ ${ }^{1}$ Department of Electrical and Electronic Engineering, Yasar University, Izmir, Turkey \\ *corresponding author, E-mail: mustafa . secmen@yasar.edu.tr
}

\begin{abstract}
In this study, the design and implementation of $\mathrm{a} \pm 45^{\circ}$ dual polarized base station antenna with improved crosspolarization discrimination (XPD) values are presented. In order to increase the cross polarization, parasitic elements are added to antenna design, which are formed by two orthogonal dipoles above ground plane. The overall antenna and radiator sizes are diminished by using meandered dipoles. The designed compact antenna has VSWR of 2:1 within 1.71-2.69 GHz frequency band, which covers GSM $1800 / 3 \mathrm{G} / \mathrm{LTE}$ bands. In the given bandwidth, minimum gain of the designed antenna is $0 \mathrm{dBi}$ within the beamwidth of $120^{\circ}\left( \pm 60^{\circ}\right)$ at azimuth plane $\left(\phi=0^{\circ}\right)$. Minimum XPD value of $2 \mathrm{~dB}$ is achieved between 1.71-2.4 GHz for $\pm 60^{\circ}$ beamwidth without parasitic elements where XPD values are improved to minimum $10 \mathrm{~dB}$ by using thick parasitic elements. The designed antenna is manufactured where return losses for both port 1 and port 2 are measured to be higher than $10 \mathrm{~dB}$, and the isolation between ports is more than $20 \mathrm{~dB}$. In addition, the measured peak gain values are varied between $3 \mathrm{~dB}$ and $7 \mathrm{~dB}$ in frequency range of 1.71 $\mathrm{GHz}-2.69 \mathrm{GHz}$. XPD values are measured better than almost $10 \mathrm{~dB}$ in the given frequency band.
\end{abstract}

\section{Introduction}

Mobile communication technologies have been rapidly developing. Depending on the mentioned development, new antenna types have been arising or performances of the existing antennas have been increasing. Especially, base station antennas become important elements in these improvements. At the earlier period of mobile communication, GSM signals were carried with only 900 and $1800 \mathrm{MHz}$ frequencies. However, nowadays, mobile communication is widely used at the frequency bandwidth of $1700-2700 \mathrm{MHz}$ with the brand 4G technology. Therefore, most of the mobile phones, which are currently used, need to cover the listed frequency bands of such as GSM (800, 900, 1800, and $1900 \mathrm{MHz}$ bands), LTE (1.8, 1.9, 2.1, $2.5 \mathrm{GHz}$ bands). For this purpose, operating frequency bandwidth of the antennas used in base stations is aimed to be between $1.7 \mathrm{GHz}$ and $2.7 \mathrm{GHz}$. In order to satisfy this kind of wide frequency bandwidth, novel wideband design techniques are implemented.
Diversity techniques are widely used in modern mobile communication systems. The fundamental benefits of the diversity technique can be listed as reducing the effect of multipath propagation and increase in the quality of signal reception. Two antennas, which are usually perpendicular to each other for the purpose of polarization diversity, are placed to give $\pm 45^{\circ}$ dual polarization [1]. At least three antennas are placed in the base stations for all space coverage in different geographical and residential scenarios (such as city, suburban and rural areas). Depending on the application and the number of antennas placed on these antennas or an antenna array, the azimuth plane angular beamwidth values vary between 65 and 120 degrees where optimum elevation beamwidth is generally selected as 7 degrees. The azimuth angular beamwidth is usually $65^{\circ}(\theta=$ $\left.\pm 32.5^{\circ}\right)$ in the city area whose base stations need more than three antennas, while the beamwidth of $120^{\circ}\left(\theta= \pm 60^{\circ}\right)$ is usually observed at the base stations in the rural area where three antennas can be enough to cover azimuth plane [2]. In base station applications, the cross polarization discrimination (XPD) between two antennas, which are generally $\pm 45^{\circ}$ dual polarized antennas in polarization diversity technique, is a fundamental parameter. Some countries can apply various regulations such as the XPD value is at least $20 \mathrm{~dB}$ along the boresight in the azimuth plane along the frequency band and at least $10 \mathrm{~dB}$ in the desired beamwidth. In general, two standard dipole antennas, which are placed perpendicular to each other, can provide $\pm 45^{\circ}$ dual polarization. Although higher than 9.5 $\mathrm{dB}$ XPD value can be obtained by using the perpendicular two dipole antennas within the beamwidth of $\theta= \pm 60^{\circ}$, at the azimuth plane $\left(\phi=0^{\circ}\right.$ plane $)$, it is only valid at a single frequency. This value is practically low, and it drops quickly when the frequency is outside the narrow frequency band around the center frequency. In literature, there are different types of $\pm 45^{\circ}$ dual polarization antennas for base stations (dipole, patch, slit), however; the majority of these studies do not give any results regarding to XPD values [39]. Besides, some of these studies, which are also focused on XPD performance in a narrow frequency band, present only XPD values on boresight of azimuth plane or over the principle planes (E-plane and H-plane) of each antenna in antenna pairs [10-13]. Only a couple of studies [14-17] shows XPD performance over the azimuth plane within 
wideband frequency and within the beamwidth of $\theta= \pm 60^{\circ}$. The studies [15] and [17] have significantly large radiators making the practical usage of the antenna in array difficult, and the studies [14] and [16] have not enough bandwidth to cover all desired mobile communication frequencies. For this purpose, the main motivation in this study is to design a $\pm 45^{\circ}$ dual polarized base station antenna, which provides

- Wide frequency band of $1.71 \mathrm{GHz}-2.69 \mathrm{GHz}$

- Low reflection loss

- High isolation between ports

- Improved cross-polarization discrimination

- Wide beamwidth

- Moderate gain

- Compact overall size and small radiator size

As the initial design, there are two horizontal microstrip straight dipoles printed on subtrates. The substrates (so the dipoles) are aligned perpendicular to each other above a ground plane. The dipole pair on the antenna is fed a microstrip (Robert's) balun feed structure. In order to increase XPD values to appropriate values, image theory, vertical parasitic elements are added to the antenna by utilizing from image theory. It is observed that thicker parasitic elements results in wider bandwidth. However, since the achieved frequency band is not sufficient, meandered technique on the horizontal dipoles are employed to both reduce antenna dimensions and increase the frequency bandwidth.

In the design and simulation process, CST Microwave program is used. A parametric optimization is employed to adjust the final dimensions on the antenna to get a frequency band between $1.71 \mathrm{GHz}$ and $2.69 \mathrm{GHz}$ frequencies. By using these parameter values, the antenna is simulated, produced and measured.

\section{Antenna Design}

\subsection{Design Theory and Preliminary Designs}

When two horizontal straight dipole antennas are configured along $\phi=-45^{\circ}$ and $45^{\circ}$ for the axes given in Figure 1, the theoretical cross-polar discrimination (XPD) values on the azimuth plane $\left(\phi=0^{\circ}\right.$ plane in Figure 1) are infinite at boresight and about $9.5 \mathrm{~dB}$ at $\theta= \pm 60^{\circ}$ when there is no ground plane. The addition of ground plane results in image dipoles below ground plane according to the image theory [18]. The current of these image dipoles flow in opposite direction with the original dipoles in Figure 1.

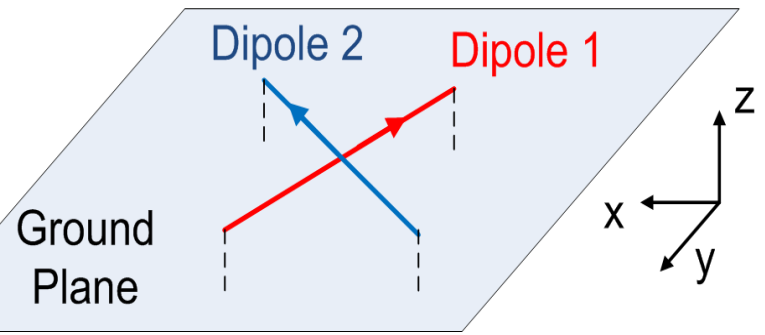

Figure 1: The configuration of two horizontal straight dipoles above ground plane.
The original horizontal dipoles along with image horizontal dipoles make $E_{\theta}$ (theta component of electric field) pattern wider and $E_{\phi}$ (phi component of electric field) pattern narrower at $\phi=0^{\circ}$ plane. Therefore, especially at the elevation angles moderately away from boresight at $\phi=0^{\circ}$ plane, $E_{\theta}$ and $E_{\phi}$ magnitudes can be closer as compared to the case without ground plane. The XPD values at these elevation angles can increase at these elevation angles with the proper selection of height values above ground plane. However, this fact is only valid at a narrow band around resonant frequency of dipoles. Besides, the XPD values within wide beamwidth such as $\theta= \pm 60^{\circ}$ may exceed just 10 $\mathrm{dB}$ practically. Therefore, in order to increase the XPD values, vertical parasitic elements are placed at the points of the dash straight lines on Figure 1. By using the image theory again, these vertical elements (monopoles), which are excited from horizontal dipoles via coupling mechanism without any electrical connections with horizontal dipoles, form vertical dipoles. These vertical dipoles theoretically make no contribution on $E_{\phi}$ pattern at $\phi=0^{\circ}$ plane, and have slight effects on $E_{\theta}$ pattern at around boresight. However, they provide significant field addition to $E_{\theta}$ pattern at the elevation angles far away from boresight. Therefore, difference between the magnitudes of $E_{\theta}$ and $E_{\theta}$ components at these far away elevation angles becomes much smaller. This effect significantly increases XPD values at these angles; consequently, improves XPD performance within much wider beamwidth. By considering explained theory for the improvement of XPD performance above, an antenna as shown in Figure 2 is initially designed and realized.

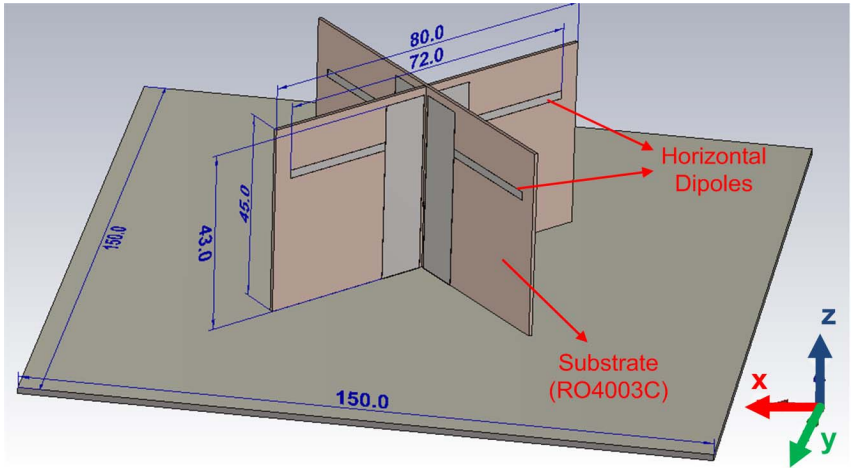

(a)

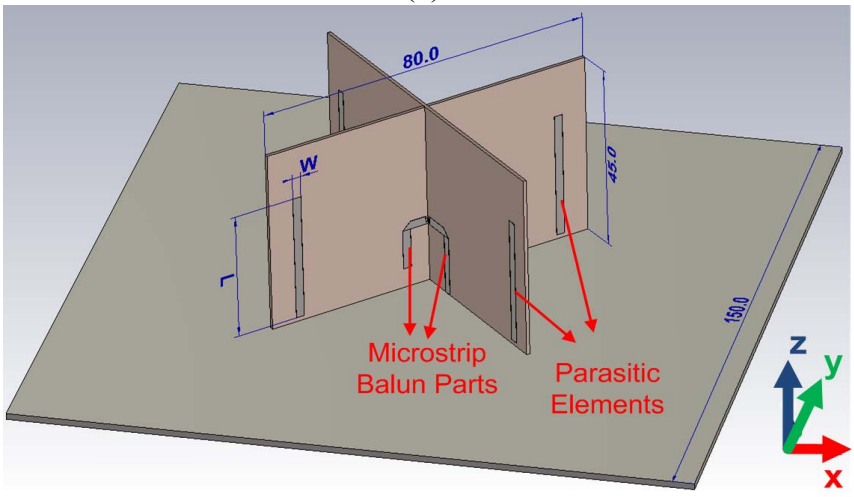

(b)

Figure 2: The views of the designed antenna structure with the dimensions in mm (a) Front view (b) Back view. 
As shown in Figure 2, all parts/elements (horizontal dipoles, parasitic elements and microstrip balun parts) are implemented on a planar dielectric substrate. The dielectric substrate is selected as Rogers $4003 \mathrm{C}$ with thickness of $\mathrm{h}=$ $0.813 \mathrm{~mm}$, dielectric constant of $\varepsilon_{\mathrm{r}}=3.55$ and tangent loss of $\tan \delta=0.0027$. The horizontal dipoles on the structure are fed by well-known microstrip (Robert's) balun as shown in Figure 3. The dimensions of the balun structure given in Figure 3 are optimized and kept constant in the following design steps/antenna versions.
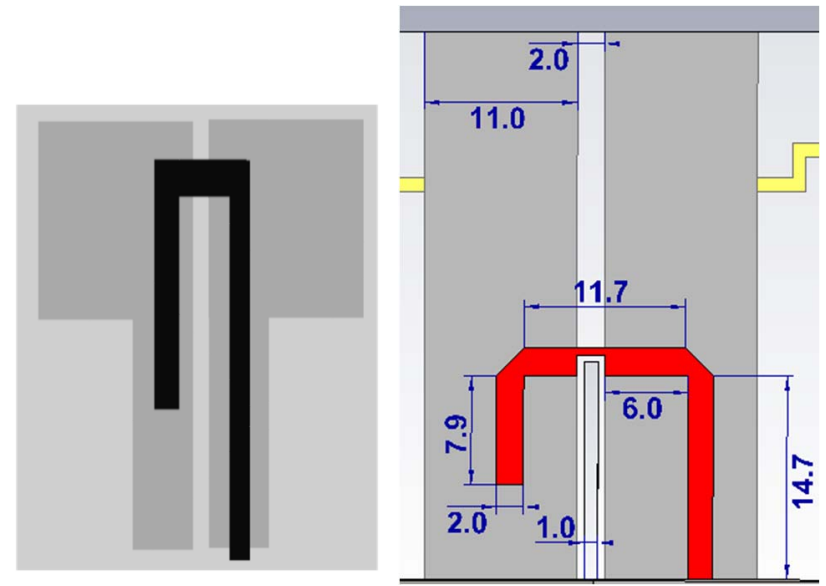

Figure 3: Robert's balun [2] and the optimized dimensions of Robert's (feed) balun of designed antenna in this study.

The horizontal dipoles in the structure are taken halfwavelength dipoles with thickness of $2 \mathrm{~mm}$. The parasitic elements are treated as quarter wavelength monopoles with height (length) of L. However, they do not touch the ground plane that they are elevated $1 \mathrm{~mm}$ above the ground plane; consequently, the bottom edges of horizontal dipoles are $(\mathrm{L}+1 \mathrm{~mm})$ above PEC ground plane. Since the antenna is desired to operate within a broadband of 1.71-2.69 GHz, the resonant frequencies of dipole and monopole structures are selected at different values. In order to cover 1.71-2.69 GHz, former's dimension is selected to give resonant at about 2.1 $\mathrm{GHz}$ and latter dimension is arranged to have resonant frequency around $2.4 \mathrm{GHz}$. Therefore, the dipole length is selected as $72 \mathrm{~mm}$, and the length (height) of the monopole is calculated as $\mathrm{L}=31 \mathrm{~mm}$ in Figure 2 . All other dimensions given Figure 2 are again calculated from optimization process carried out in CST Microwave Studio 2017. In the preliminary designs, in order to observe the effects of dimensions/positions of parasitic elements on the return loss performance, several trials have been realized. As the initial trial, the thickness (width) of the parasitic element is selected as $\mathrm{W}=2 \mathrm{~mm}$. The position of these parasitic elements is arranged with respect to horizontal dipole such that the center of upper edge of the vertical parasitic element almost has same radial distance with edge of the horizontal dipole as shown in Figure 4(a). The return loss and isolation performances of the antenna with configuration in Figure 4(a) are shown in Figure 5. It can be observed from the results in Figure 5 for $1.71-2.69 \mathrm{GHz}$ that although the isolation performance of the antenna is adequate (being more than $30 \mathrm{~dB}$ ), the return loss performance is not enough.

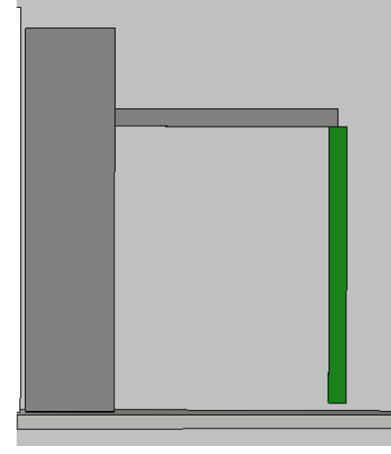

(a)

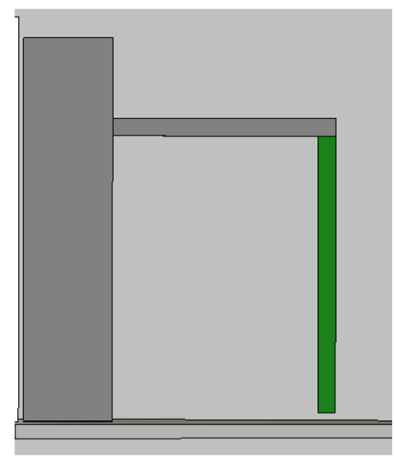

(c)

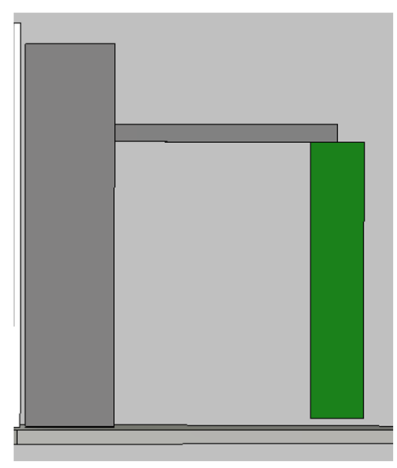

(b)

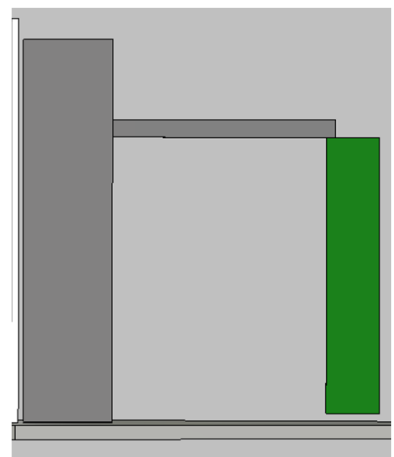

(d)
Figure 4: Different positions and width values (different trials) of each parasitic element in the simulations of the proposed antenna. (a) $\mathrm{W}=2 \mathrm{~mm}$ (b) $\mathrm{W}=6 \mathrm{~mm}$ (c) $\mathrm{W}=2$ $\mathrm{mm}$ and shifted inward direction (d) $\mathrm{W}=6 \mathrm{~mm}$ and shifted outward direction. Here, vertical (green) parasitic element is on one side while horizontal (gray) dipole arm is on the other side of the substrate.

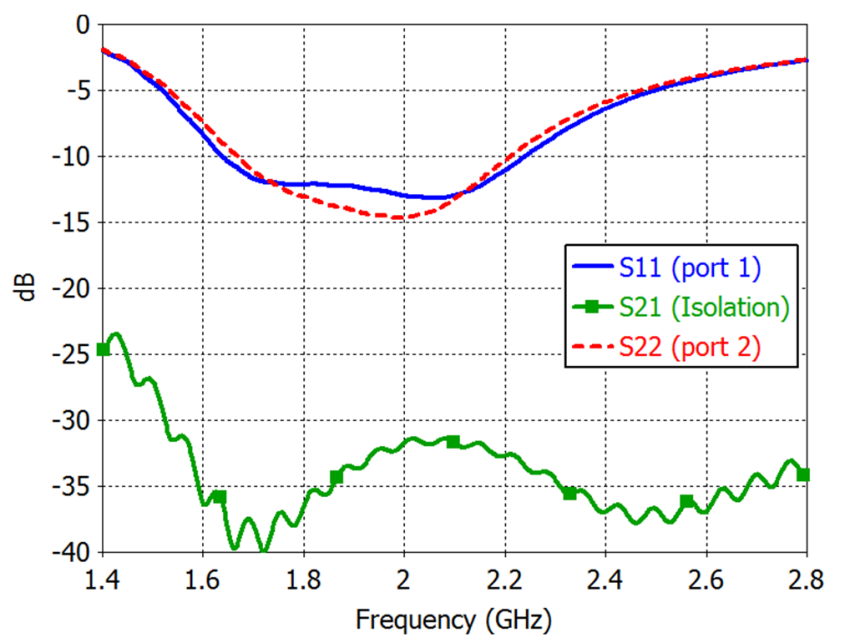

Figure 5: The reflection coefficients of each port and isolation between ports for the antenna with the configuration depicted in Figure 4(a).

Due to the insufficient performance which provides 1.7-2.2 $\mathrm{GHz}$ frequency band even for $10-\mathrm{dB}$ return loss, different trials by changing width and position of identical parasitic elements are performed. As shown in Figure 4, these modifications are the use of thicker parasitic elements or the shift of these elements in radial direction on the substrate. 


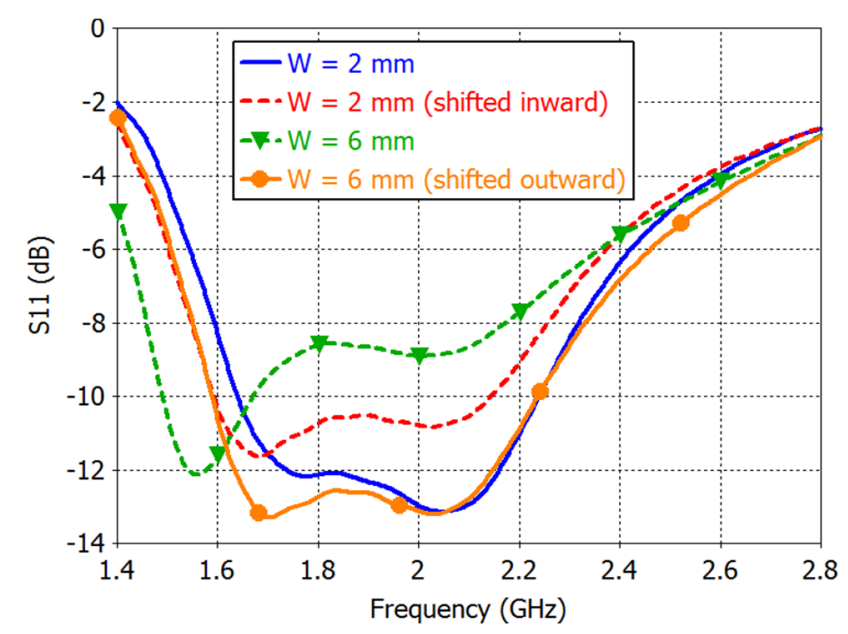

Figure 6: The reflection coefficient values of port 1 of the antenna with all configurations in Figure 4.

In the simulations of new trials (designs), all the parameter values except parasitic width and position are kept constant. The return loss performances only for port 1 are demonstrated in Figure 6 for the simplicity where port 2 is also found to show similar characteristics. The reflection coefficient curves in Figure 6 reveal some crucial results to observe the effect of parasitic element on the return loss. First of all, shifting the parasitic element in radially inward direction is found to give worse return loss performance. This is due to the fact the feeding mechanism of the parasitic element from horizontal dipole is disturbed more when parasitic element is further away from the edge of the dipole. This effect can be also observed for the case with $\mathrm{W}$ $=6 \mathrm{~mm}$ and no shift in Figure 4(b) (whose results are given with triangle marks and green and dashed curve in Figure 6) such that some portion of the parasitic element extends along inward direction resulting in disturbance in coupling mechanism. In addition, it is also obtained that the thicker parasitic element with the extension along outward direction provides wider return loss bandwidth as given in circle marks and orange and solid curve with $\mathrm{W}=6 \mathrm{~mm}$ in Figure 6. However, even for this case, the bandwidth for $\left|S_{11}\right|<-10$ $\mathrm{dB}$ is about $0.65 \mathrm{GHz}$ (between 1.6-2.25 GHz), which is not sufficient. Therefore, parasitic elements should be much thicker than $\mathrm{W}=6 \mathrm{~mm}$ and should extend along radially outward direction to get desired bandwidth of about $1 \mathrm{GHz}$. When the minima of the reflection coefficients are examined, it can be stated that there are two resonant frequencies: one being steady at almost $2.1 \mathrm{GHz}$ and the other decreasing from $1.8 \mathrm{GHz}$ for different variations of the parasitic elements. The frequency of $2.1 \mathrm{GHz}$ belongs to the length of the horizontal dipole, which is consistent with the mathematical (theoretical) expectation. The stable result for this frequency is also reasonable since the horizontal dipoles are kept constant for different trials of parasitic elements. On the other hand, varying resonance frequency in the range of 1.6-1.8 GHz belongs to different variations of the parasitic elements. As again expected, wider parasitic elements give lower resonance frequency where similar effect is observed in monopole or dipole antennas [18]. However, resonant frequency around $1.7 \mathrm{GHz}$ deviates moderately with the theoretical expectation of about 2.4 $\mathrm{GHz}$ by considering height of the monopole. This deviation is caused by dielectric loading effect of the substrate and capacitance effect between lower edge of the parasitic element and the ground plane.

\subsection{Meandered Line Modification and Final Design}

After the parametric study employed in Section 2.1 and the substantial conclusions acquired from the results, some modifications are done to obtain desired frequency band of 1.71-2.69 GHz. With the idea of usage of thick structures as being one of the methods to increase the bandwidth [19], it is needed parasitic elements with much thicker than $\mathrm{W}=6$ $\mathrm{mm}$ to get about $1 \mathrm{GHz}$ band. However, if the thickness is extended along outward direction, the total width of the dielectric substrate (given as $80 \mathrm{~mm}$ in Figure 2) should be increased accordingly. However, it results in a significant increase in radiator size, which is undesired property in base station arrays. Therefore, in order to extend the parasitic element along inward direction, it is decided to decrease horizontal length of the dipoles in the structures. The shortening of horizontal dipoles is also needed to increase the resonant frequency of horizontal dipole from $2.1 \mathrm{GHz}$ to higher values in order to make effective coverage of the frequency band of 1.71-2.69 GHz. Besides, since the increase in the thickness of the parasitic elements further decrease the resonance frequency below $1.6 \mathrm{GHz}$, the length (L) of the parasitic elements is also reduced.

Even with the modifications of the shortened straight dipoles and shortened and thickened parasitic elements, 10$\mathrm{dB}$ return loss band for 1.71-2.69 $\mathrm{GHz}$ could not be achieved with optimization/parametric studies performed in CST when the width of the substrate is fixed to $80 \mathrm{~mm}$ such that the mentioned band can be obtained with larger substrate width values, which causes increase in the radiator size of the antenna. In order to keep the radiator size of the antenna as low as possible (consequently to make the antenna as compact as possible), straight horizontal dipoles are replaced with meandered line dipoles. Meander structure, whose design procedures and other relevant information are given in [20] and [21] in detail, is one of the main structures used in the printed antennas to reduce the overall (edge-to-edge) dimension of the monopole/dipole antennas. By using the meandered dipoles as the final modification in the design, end-to-end horizontal dimension of the dipoles is decreased without changing the resonant frequency contributed from dipoles. However, the reduction in the horizontal dimension of the dipoles allows additional extension in the thickness of parasitic elements along radially inward direction instead of outward direction. Therefore, desired bandwidth enhancement can be achieved without increasing the width of the dielectric substrate or the size of the radiators. The antenna structure given in Section 2.1 is optimized again by using shorter but thicker parasitic elements and meandered line dipoles. In the optimization, all parameters including the dimensions of other parts (apart from parasitic elements and dipoles) given in Figure 2 are also used. The dimensions of the final design of the proposed antenna in $\mathrm{mm}$ are shown in Figure 7. 


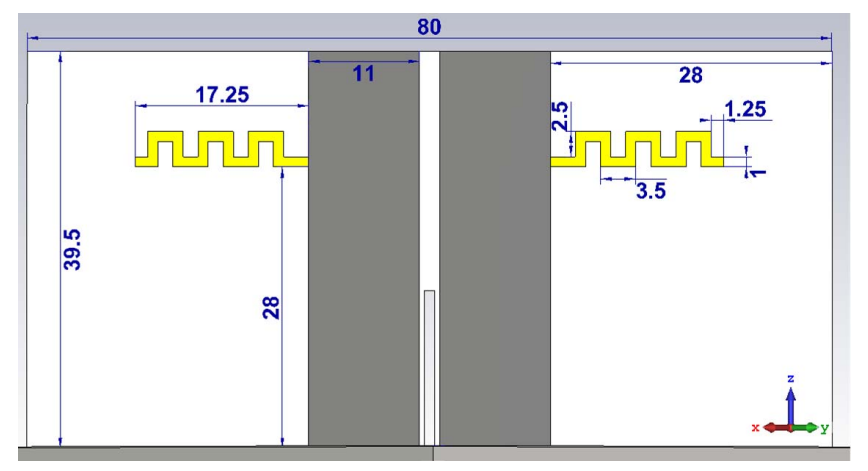

(a)

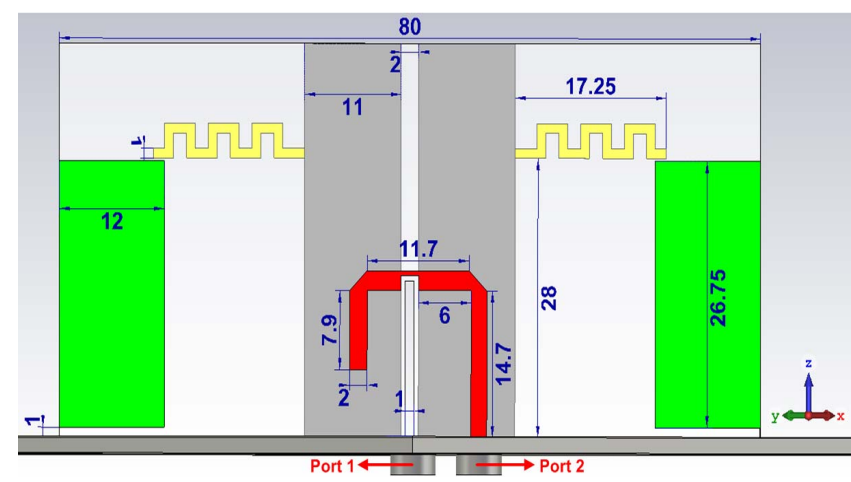

(b)

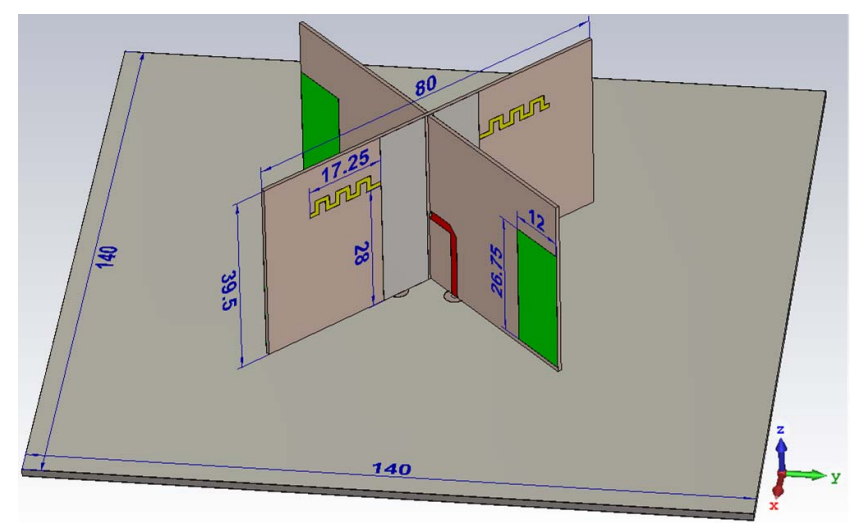

(c)

Figure 7: (a) Front side for one of the substrates (b) Back side for one of the substrates where parts with shaded colors belong to front side (c) Perspective view of overall antenna including ground plane. All units are in millimeters.

As it can be seen from views in Figure 7 , since the horizontal length of meandered lines is $17.5 \mathrm{~mm}$, which gives about $59 \mathrm{~mm}$ edge-to-edge horizontal distance for dipoles, it is possible to insert thicker parasitic elements in the final design. The height of the substrate (or the height of overall antenna) is also reduced since shorter parasitic elements are employed. Therefore, a reduction in height of the antenna is achieved as compared to preliminary designs that the goal of compactness in size is satisfied. Besides, ground plane with $4 \mathrm{~mm}$ thickness has lower dimensions of $140 \mathrm{~mm}$ by $140 \mathrm{~mm}$ corresponding to almost $\lambda_{0}$ by $\lambda_{0}$ at the center frequency of $2.2 \mathrm{GHz}$ of the desired band. Besides, the radiator size is obtained as $80 / \sqrt{ } 2=56.35 \mathrm{~mm}$ by $80 / \sqrt{ } 2$ $=56.35 \mathrm{~mm}\left(\lambda_{0} / 2\right.$ by $\lambda_{0} / 2$ at highest frequency of $\left.2.7 \mathrm{GHz}\right)$.

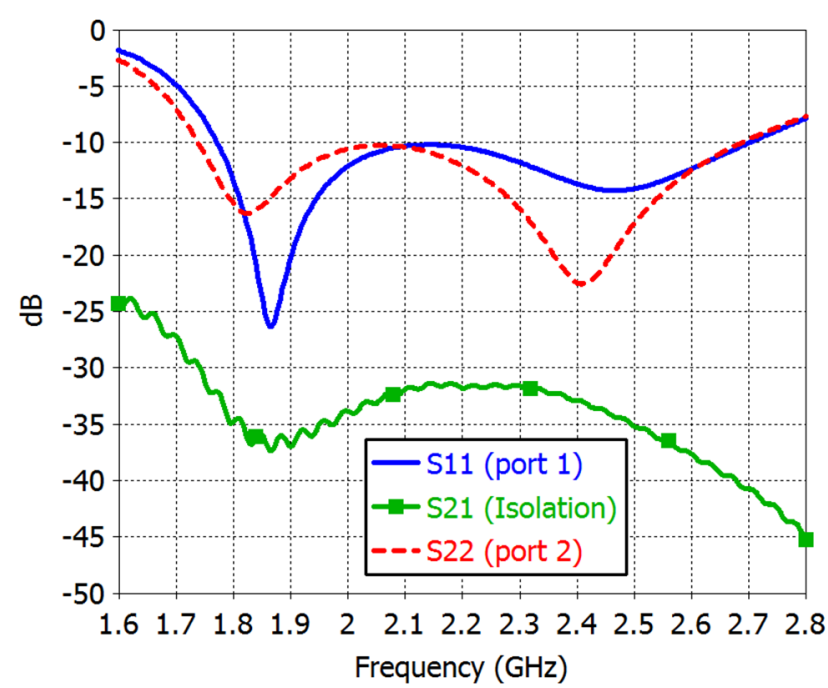

Figure 8: The simulation results of final design of the proposed antenna for reflection coefficients and isolation.

The return loss and isolation performances of the final design of the proposed antenna are demonstrated in Figure 8. From the results given in Figure 8, the mentioned antenna gives more than almost $10 \mathrm{~dB}$ return loss for each port and higher than $30 \mathrm{~dB}$ port-to-port isolation within frequency band of 1.75-2.69 GHz. The band for 10-dB return loss of port 1 is slightly shifted to the right as compared to port 2 that the lower frequency of port 1 is approximately 1.75 $\mathrm{GHz}$. Therefore, the return loss and isolation performances of the antenna are found to be sufficient from the realized simulations where the other performances will be given in the next section as well as the comparison with the antennas without parasitic element and with straight dipoles instead of meandered line dipoles.

\subsection{Performance Comparison of the Simulation Results}

After the design of the proposed antenna is finalized, the performances of the antenna (to be called as antenna 1) are compared with the antenna without parasitic elements (antenna 2) and the antenna with straight dipoles and thin, long parasitic elements (antenna 3). The results of the antenna without parasitic elements are compared to represent the superiority of the usage of these elements. For the antenna without parasitic elements, the simulations are repeated by just removing parasitic elements in the antenna in Figure 7 and keeping other dimensions same. For antenna 3 with straight dipoles and thin, long parasitic elements, the simulation results performed for the antenna given in Figure 2, Figure 3 and Figure 4(a) are considered. The simulation results of the antenna with and without parasitic elements are given in Figure 9 for reflection coefficients and isolation. For the results of reflection coefficients in Figure 9(a), it is clear that the parasitic elements have significant improvement on the return loss performance. The bandwidth of 10-dB return loss for both ports of the antenna without parasitic elements are much narrower such that the frequency band is about $2.5-2.8 \mathrm{GHz}$ by considering two ports while the mentioned band is between 1.75-2.69 GHz for antenna with parasitic elements. 


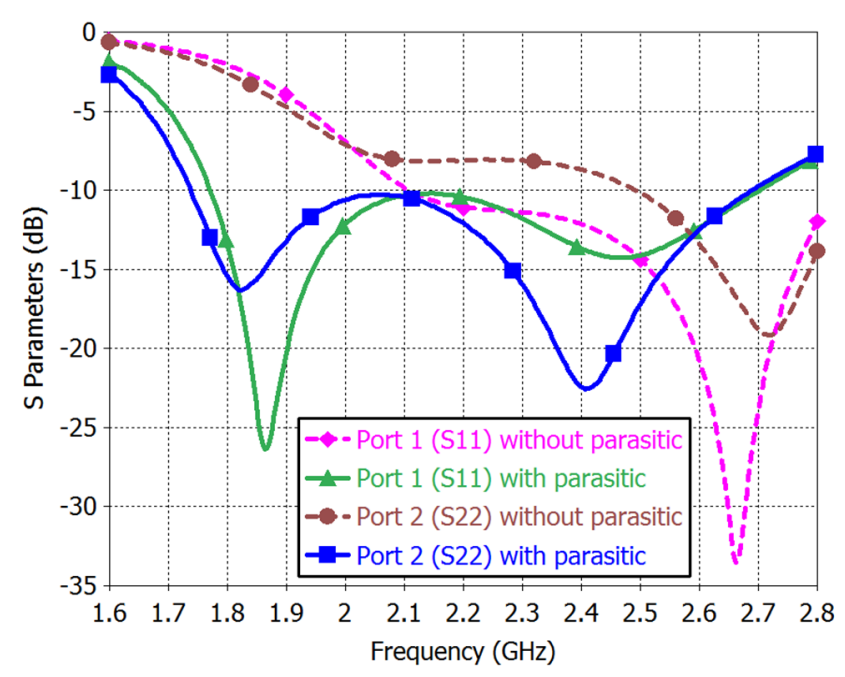

(a)

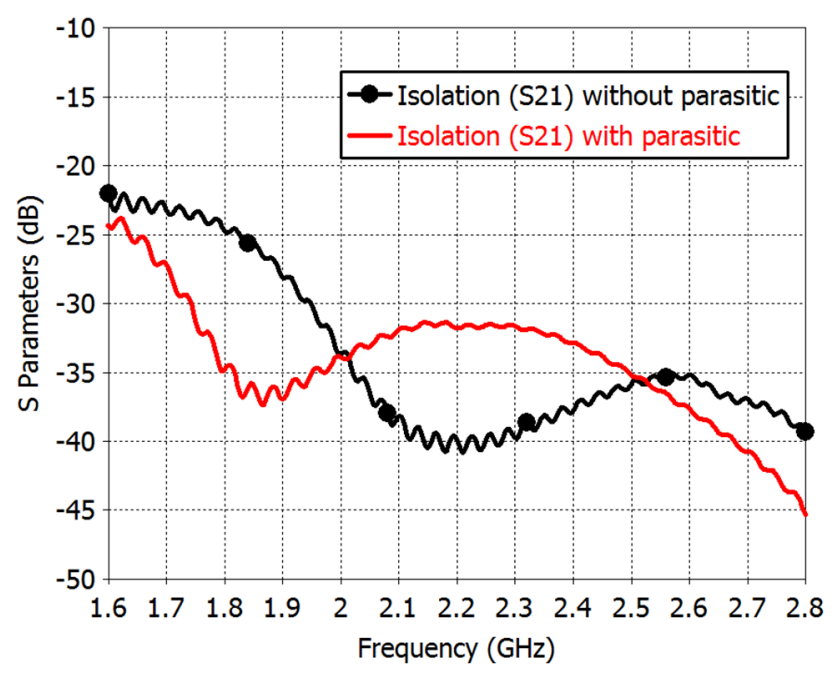

(b)

Figure 9: The comparison of simulation results of the proposed antenna with and without parasitic elements for (a) return loss of each port (b) isolation.

The effect of parasitic elements can be also understood from the resonance frequencies in Figure 9(a). These elements add a resonance frequency on $1.8 \mathrm{GHz}$ and also shift the resonance frequency belonging to horizontal dipole to approximately $2.4 \mathrm{GHz}$. Therefore, two resonance frequencies provide wide bandwidth. On the other hand, the antenna without parasitic elements has only one resonance frequency around $2.6 \mathrm{GHz}$, which results in narrow band. When the port-to-port isolation performances in Figure 9(b) are handled, the antenna with parasitic elements also has better performance by giving more than $30 \mathrm{~dB}$ isolation within the band of 1.75-2.8 GHz, which is more than $23 \mathrm{~dB}$ for the antenna without parasitic elements in the same frequency band. The relevant performances of the antenna with straight dipoles (antenna 3) are not put in Figure 9 since they were given in Section 2.1. Although the isolation performance of this antenna is similar to the antenna in Figure 7 , the return loss bandwidth is again narrower (between 1.7-2.2 GHz).
After the return loss and isolation performances are compared, the peak gain values are compared. As being one of the possible drawbacks of the proposed antenna, although the usage of meandered lines instead of straight lines can provide reduction in total length, it can cause drop in the antenna gain $[20,21]$. Besides, the usage of thick vertical elements may contribute to gain reduction mechanism. Therefore, the gain values of proposed antenna (antenna 1) and the antenna with straight dipoles and thin parasitic elements (antenna 3) are obtained in order to observe whether the usage of meandered lines and thick parasitic elements brings considerable reduction in the gain or not. The peak gain values of antenna 1 and antenna 3 at some frequencies are given in Table 1. Here, co-pol peak gain values at azimuth plane $\left(\phi=0^{\circ}\right.$ plane with respect to axes given in Figure 2) are only given where the values for elevation plane $\left(\phi=90^{\circ}\right.$ plane in Figure 2$)$ are similar.

Table 1: Comparison of simulated peak gain values for antenna 1 and antenna 3 at azimuth plane $\left(\phi=0^{\circ}\right)$.

\begin{tabular}{ccccc}
\hline & \multicolumn{2}{c}{ Port 1 } & \multicolumn{2}{c}{ Port 2 } \\
\hline Frequency & Ant. 1 & Ant. 3 & Ant. 1 & Ant. 3 \\
{$[\mathrm{GHz}]$} & {$[\mathrm{dBi}]$} & {$[\mathrm{dBi}]$} & {$[\mathrm{dBi}]$} & {$[\mathrm{dBi}]$} \\
1.8 & 7.30 & 7.21 & 7.31 & 7.26 \\
2 & 6.34 & 6.55 & 6.05 & 6.62 \\
2.2 & 4.42 & 4.67 & 4.40 & 4.58 \\
2.4 & 3.55 & 2.76 & 3.54 & 2.71 \\
2.6 & 2.96 & 0.96 & 2.83 & 0.9 \\
\hline
\end{tabular}

When the results in Table 1 are examined, it can be stated that although the peak gain values of antenna 3 are slightly higher than those of antenna 1 at lower frequencies up to $2.4 \mathrm{GHz}$, the gain performance of antenna is better at higher frequencies in the band. Besides, it is shown that the meandered lines and thick parasitic elements do not cause significant gain drop where antenna 1 has at most $0.5 \mathrm{~dB}$ lower gain values. The proposed antenna is found to give between $2.6 \mathrm{dBi}$ and $7.3 \mathrm{dBi}$ gain within the frequency band of 1.71-2.69 GHz. When it is considered that base station antennas are generally used in arrays, which provides larger ground plane, the single element realized gain of the proposed antenna will probably increase when used in array. The $3-\mathrm{dB}$ beamwidth values of antenna 1 are found to be minimum 78 degrees. Therefore, the proposed antenna can approximately give at least $13 \mathrm{dBi}$ peak gain when it is used in $11 \times 1$ array in order to obtain optimum elevation beamwidth of 7 degrees described in Section 1 .

As the final comparison, the gain and XPD performances of the antennas are handled within the beamwidth of $120^{\circ}$ $\left(\theta= \pm 60^{\circ}\right)$ at azimuth plane $\left(\phi=0^{\circ}\right)$. The corresponding simulation results of all antennas for the mentioned performances are presented in Table 2. From the results for $120^{\circ}$ beamwidth and 1.71-2.69 GHz, the proposed antenna is shown to be superior to the antenna without parasitic elements in terms of minimum gain and XPD. The minimum gain and XPD increase to $0 \mathrm{dBi}$ and $7 \mathrm{~dB}$ respectively by using parasitic elements which are just -9.5 $\mathrm{dBi}$ and $2 \mathrm{~dB}$ for antenna 2 . Besides, the proposed antenna has minimum XPD of $10 \mathrm{~dB}$ for the band of 1.71-2.4 GHz, 
which is still $2 \mathrm{~dB}$ for antenna 2 . Therefore, the usage of the parasitic elements is verified to improve XPD performance.

Table 2: The comparison of gain and XPD performances of all antennas at azimuth plane $\left(\phi=0^{\circ}\right)$.

\begin{tabular}{|c|c|c|c|}
\hline & Ant. 1 & Ant. 2 & Ant. 3 \\
\hline $\begin{array}{c}\text { Minimum Gain for } \\
\text { 1.71-2.69 GHz within } \\
\text { beamwidth of } 120^{\circ} \\
{[\mathrm{dBi}]}\end{array}$ & 0 & -9.5 & -2 \\
\hline $\begin{array}{l}\text { Minimum XPD for } \\
\text { 1.71-2.69 GHz within } \\
\text { beamwidth of } 120^{\circ} \\
{[\mathrm{dB}]}\end{array}$ & 7 & 2 & 7 \\
\hline $\begin{array}{c}\text { Minimum XPD for } \\
1.71-2.4 \mathrm{GHz} \text { within } \\
\text { beamwidth of } 120^{\circ} \\
{[\mathrm{dB}]}\end{array}$ & 10 & 2 & 10 \\
\hline $\begin{array}{l}\text { Minimum Gain for } \\
\text { 1.71-2.69 GHz within } \\
\text { beamwidth of } 65^{\circ} \\
{[\mathrm{dBi}]}\end{array}$ & 0 & -2 & -2 \\
\hline $\begin{array}{l}\text { Minimum XPD for } \\
\text { 1.71-2.69 GHz within } \\
\text { beamwidth of } 65^{\circ} \\
{[\mathrm{dB}]}\end{array}$ & 10 & 12 & 10 \\
\hline
\end{tabular}

The results for the beamwidth of $65^{\circ}$, which can be the case for city area as explained in Section 1, are also given in Table 2. XPD results of antenna 1 and antenna 2 are close that the usage of parasitic elements does not considerable effect on XPD performance for $65^{\circ}$ beamwidth. This result is reasonable with the explanation given in Section 1 that the addition of vertical parasitic element is expected to improve XPD values at the elevation angles far away from boresight. Therefore, the parasitic elements are observed to give almost same XPD values up to $\theta= \pm 32.5^{\circ}$, and to improve XPD values for the elevation angles greater than $32.5^{\circ}$ (up to $\theta= \pm 60^{\circ}$ ). Consequently, the proposed antenna with parasitic elements enhances XPD performance over wider beamwidth in azimuth plane.

When the results of antenna 1 and antenna 3, where the latter still uses parasitic elements with thinner width, are considered, similar XPD performances are calculated. Therefore, it is also concluded that the thickness of vertical parasitic elements is not a crucial parameter to effect XPD improvement. Instead, it has great importance in the enhancement of the return loss bandwidth such that realized minimum gain performance of antenna 1 is found to be better than both antenna 2 and antenna 3 .

\section{Manufacturing and Measurement Results}

\subsection{Manufacturing Steps}

In order to verify the design given in Figure 7 , the proposed antenna is fabricated. The manufacturing of the antenna contains three main steps, which are the fabrication of antenna parts above ground, form of the ground plane, and the assembly of antenna parts, ground plane and SMA connectors. As described in the design of the antenna; the printed meandered dipoles, parasitic elements and microstrip balun structures are placed on the substrates, which are placed mutually perpendicular to each other and ground plane. The material of Rogers RO4003C $\left(\mathrm{h}=0.813 \mathrm{~mm}, \varepsilon_{\mathrm{r}}=\right.$ $3.55, \tan \delta=0.027$ ) given in the design is selected as a substrate in the manufacturing. As the first step of the manufacturing, the parts on $\mathrm{RO} 4003 \mathrm{C}$ are produced.

The second step of the manufacturing is about the ground plane and SMA connectors' assembly. In the design and simulations, ground plane is selected as perfect electric conductor (PEC) with the thickness of about $4 \mathrm{~mm}$. However, a copper plate with the dimensions of $140 \mathrm{~mm} \times$ $140 \mathrm{~mm} \times 4 \mathrm{~mm}$ becomes too heavy for the production and especially for pattern measurement systems. Therefore, in the ground plane production, pure copper plate is replaced with the plates of FR4 material having nearly $35 \mu \mathrm{m}$ copper thickness values on each side in order to get much lighter antenna in weight. In order to get suitable ground thickness at approximately $4 \mathrm{~mm}$, two pieces of double-sided FR4 substrate each having $1.65 \mathrm{~mm}$ total thickness are connected to each other and soldered. Thus, a ground plane having almost $3.3 \mathrm{~mm}$ thickness is constituted that the manufactured antenna becomes lighter and more compatible with the measurement setup. The ground plane is drilled with $0.635 \mathrm{~mm}$ radius, and inner parts of SMA connectors are passed through the ground. Then, SMA connectors are soldered to the microstrip balun and ground. As being third and final step, antenna parts are soldered and combined with ground and SMA connectors. Finally, the realization of the antenna is completed as depicted in Figure 10.

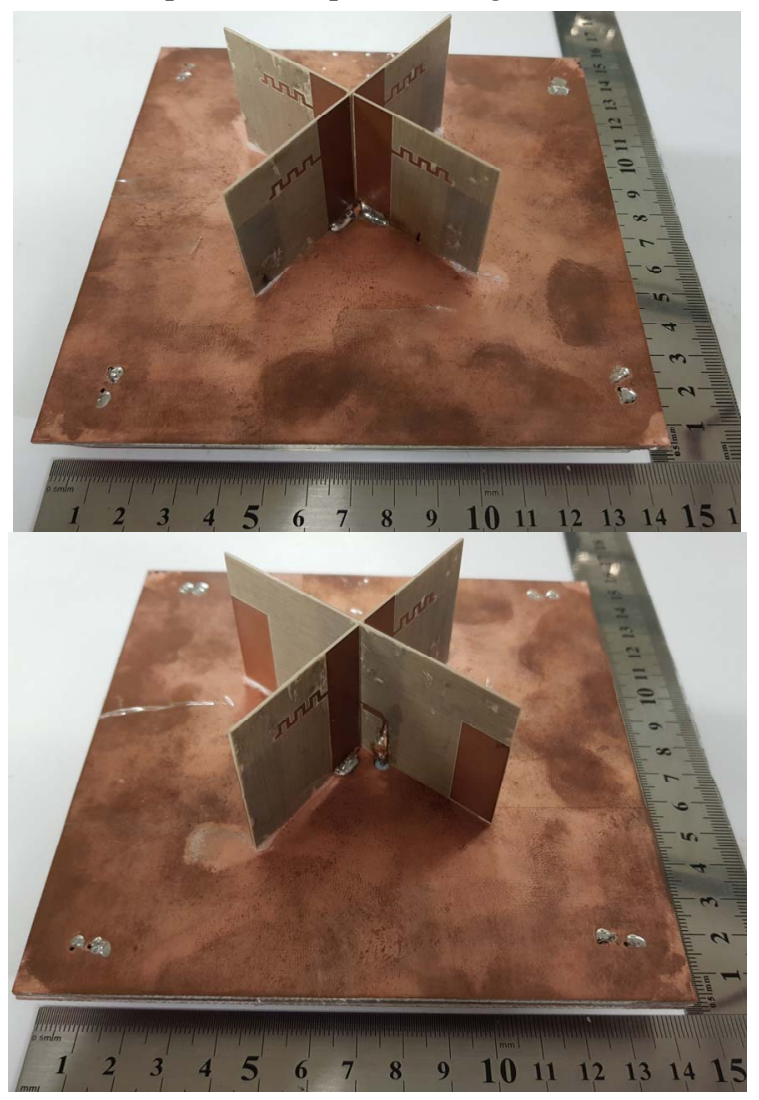

Figure 10: The photographs of the manufactured antenna. 


\subsection{Measurement Results and Comparison with Simulation Results}

After the manufacturing steps of the antenna given in Section 3.1 are completed, the performances (return loss, isolation, gain, XPD and radiation patterns) of the fabricated antenna are measured. All measurements are carried out in Antenna and Microwave Laboratory of Yasar University. The measurement of S-parameters (return loss and isolation) is done with Keysight N9912A Fieldfox RF Analyzer as shown in Figure 11(a), and radiation pattern measurements are realized with Dreamcatcher pattern measurement system [22], which includes N9912A RF analyzer as depicted in Figure 11(b).

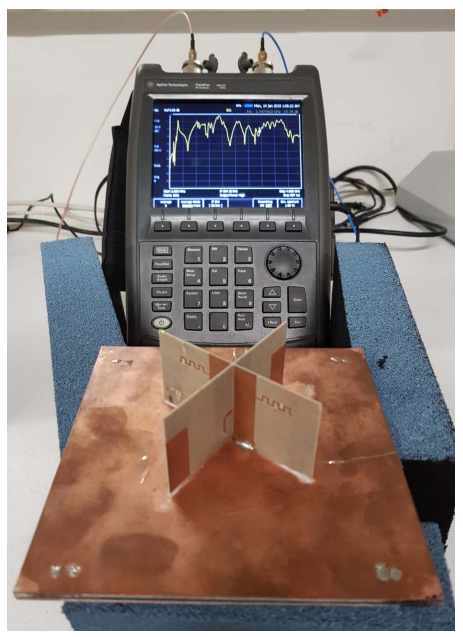

(a)

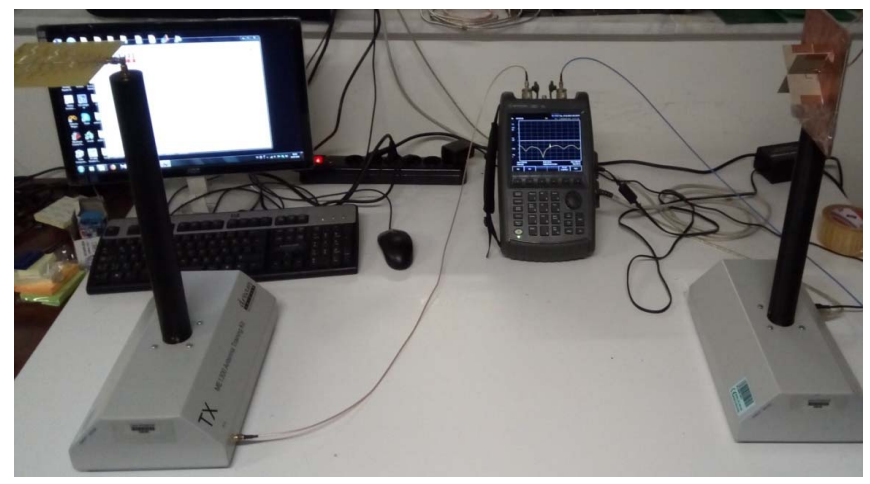

(b)

Figure 11: The photographs of the measurement setup for (a) $\mathrm{S}$ paramaters (b) Gain and radiation patterns.

The measurement results of the manufactured antenna for reflection coefficients of both ports and isolation are presented in Figure 12(a) and Figure 12(b), respectively, along with the simulation results of the same antenna given in Figure 8 for comparison. For the reflection coefficients of both ports, the measurement and simulation results are consistent that there is only about $0.1 \mathrm{GHz}$ shift between results which gives about $4 \%$ deviation. This discrepancy is possibly caused by manufacturing errors such as production tolerance, assembly or soldering errors. According to measurement results, the proposed antenna have $10-\mathrm{dB}$ return loss (RL) band of 1.8-2.8 GHz.

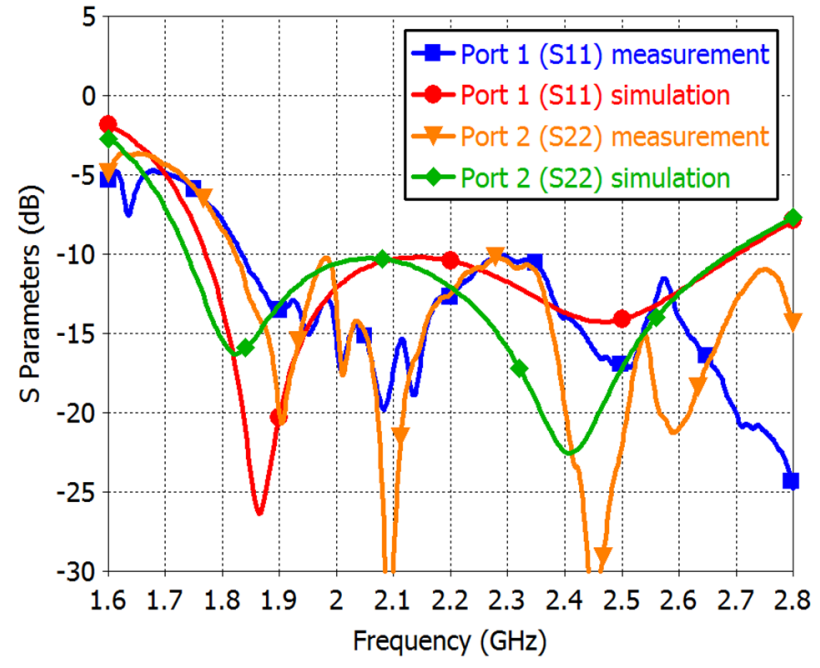

(a)

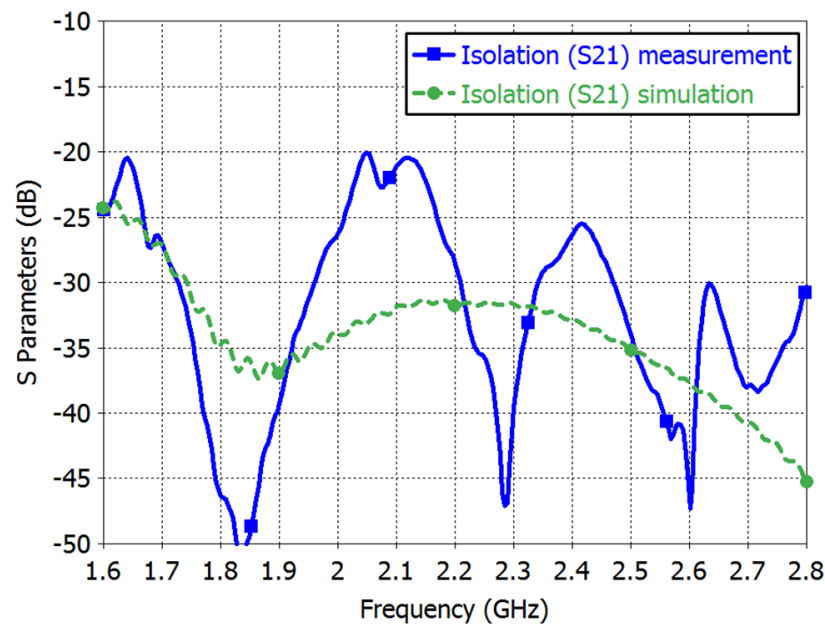

(b)

Figure 12: The comparison of measurement and simulation results of the proposed antenna for (a) return loss of each port (b) isolation.

In terms of isolation performance, the simulation and measurement results are in moderate agreement where the differences in the responses are again due to manufacturing errors. The measurement results reveal that the antenna has more than $25 \mathrm{~dB}$ port-to-port isolation at the band of 1.7-2.8 $\mathrm{GHz}$ except the region between $2.01 \mathrm{GHz}$ and $2.16 \mathrm{GHz}$ at which the isolation is better than $20 \mathrm{~dB}$. Thus, the proposed antenna is found to give higher than $20 \mathrm{~dB}$ isolation within the frequency band of 1.7-2.8 GHz.

As the next performance analysis, radiation patterns, gain, XPD values of the proposed antenna is measured. In the measurement of gain values with the technique in [15], a reference wideband antenna of Aaronia Hyperlog 60180 is also used [23]. The unnormalized gain patterns are shown in Figure 13 for two sample frequencies of $1.8 \mathrm{GHz}$ and 2.4 $\mathrm{GHz}$, which are important frequencies in base station application that former frequency belongs to GSM 1800 and latter is used Wi-Fi. Here, co-polar and cross-polar realized gain patterns of port 1 are given for both measurement and simulation where the results for port 2 are also similar. 


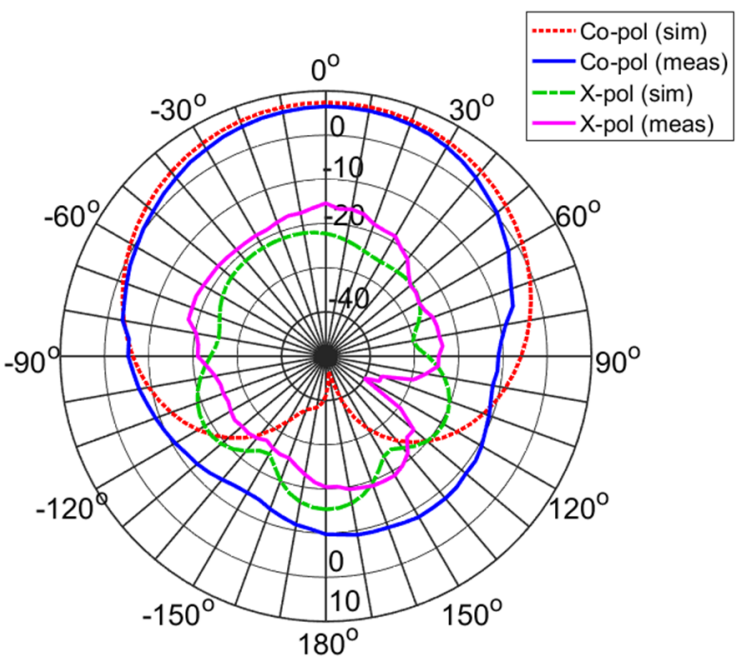

(a)

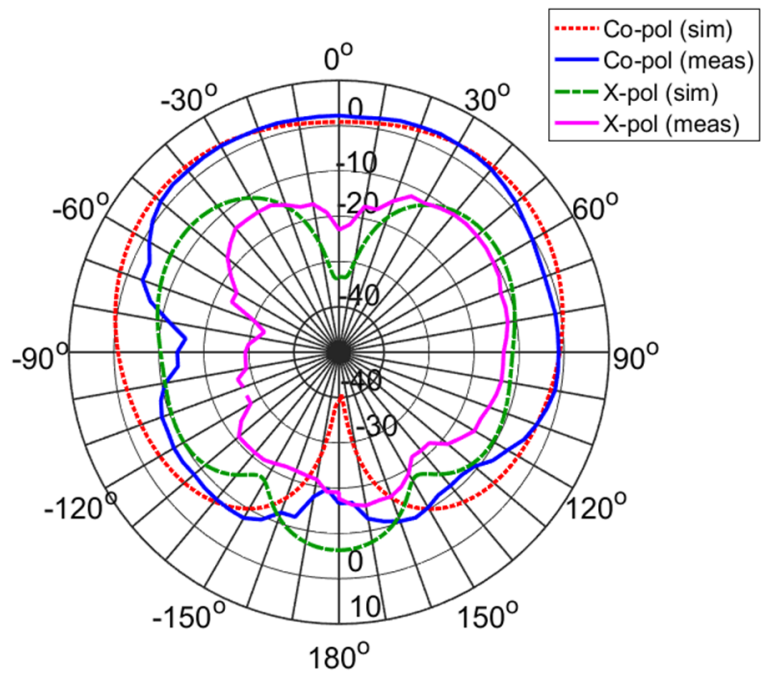

(b)

Figure 13: The measured and simulated co-polar and cross polar realized gain patterns in $\mathrm{dBi}$ for port 1 at (a) $1.8 \mathrm{GHz}$ (b) $2.4 \mathrm{GHz}$ (sim: simulation, meas: measurement).

It can be seen from the patterns in Figure 13, measured and simulated patterns match well except backlobes of the copolar patterns where backlobe gain is about $-40 \mathrm{dBi}$ in the simulations while they are just $-10 \mathrm{dBi}$ and $-20 \mathrm{dBi}$ for 1.8 $\mathrm{GHz}$ and $2.4 \mathrm{GHz}$ for the measurement, respectively. The reason of these differences is the usage of two double-sided FR4 plates for the ground plane in order to decrease the weight of the antenna as explained in Section 3.1. Therefore, the ground plane effect in measurement is not observed as strong as in simulations where full PEC plate is used for ground plane. However, backlobe level is not a significant specification considered in base station antenna due to the fact that backed metal reflectors are generally inserted between antennas placed in different sectors. Thus, backlobe level is effectively suppressed with these metal structures. Gain and radiation performances at mainlobe or especially within $120^{\circ}$ beamwidth are more crucial characteristics to notice where proposed antenna is in very good agreement with simulation results in this angle range.
The peak co-pol gain values for the patterns of port 1 are measured as approximately $6.5 \mathrm{dBi}$ and $3 \mathrm{dBi}$ at $1.8 \mathrm{GHz}$ and $2.4 \mathrm{GHz}$, respectively. When peak gain values for the simulation are examined from Table 1, these gain values are also consistent with the simulated ones. When the frequency band of 1.71-2.69 GHz is considered, the peak co-pol realized gain varies between $2.9 \mathrm{dBi}$ and $6.8 \mathrm{dBi}$.

After the measurement of gain patterns, the XPD performance of the antenna is obtained. XPD values in $\mathrm{dB}$ are extracted by taking the difference between co-pol and cross-pol gain patterns (in $\mathrm{dBi}$ ) at Figure 13. The corresponding XPD patterns for port 1 are depicted in Figure 14 within the beamwidth of $120^{\circ}\left(\theta= \pm 60^{\circ}\right)$ at the same frequencies. The XPD values are measured to have more than $20 \mathrm{~dB}$ for the angles around boresight at these frequencies. The proposed antenna provides more than 10 $\mathrm{dB}$ XPD within $120^{\circ}$ beamwidth for 1.71-2.4 GHz band, which is same result obtained from simulations as given in Table 2. When the frequency band of 1.71-2.69 GHz and both ports are considered, the antenna is measured to give at least $8.5 \mathrm{~dB}$ XPD value for $120^{\circ}$ beamwidth.

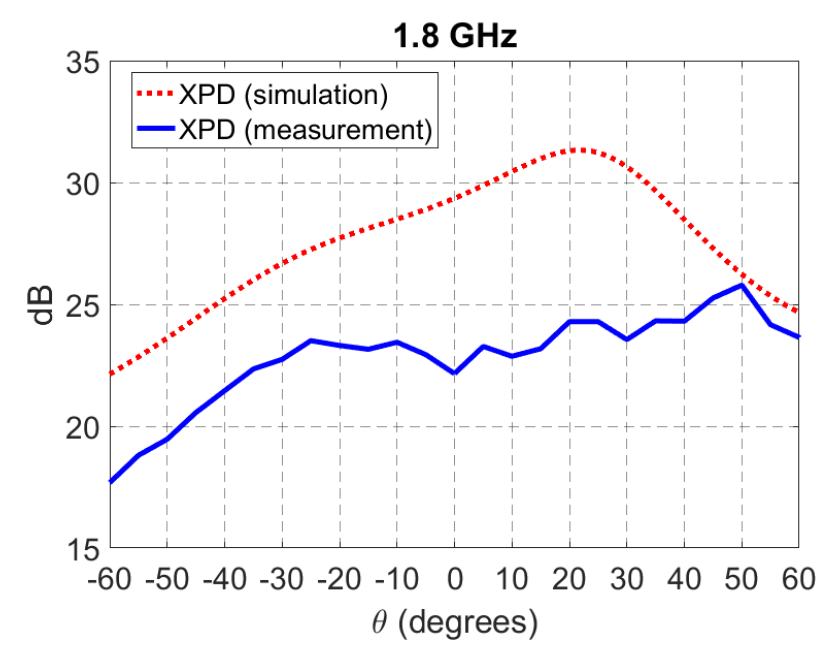

(a)

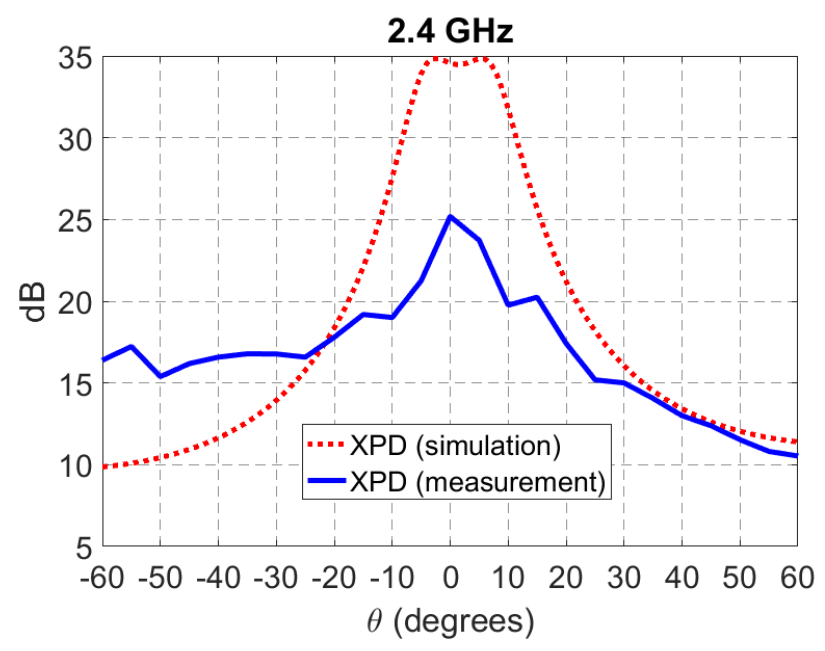

(b)

Figure 14: The measured and simulated XPD patterns for port 1 at (a) $1.8 \mathrm{GHz}$ (b) $2.4 \mathrm{GHz}$. 


\section{Discussion}

As explained in Section 1, although there are many studies about $\pm 45^{\circ}$ dual polarization antennas for base stations, only a few of these studies examines the XPD performance. In this section, the performance results of the proposed antenna are only compared with those of the studies taking XPD into consideration, and the advantages of the proposed antenna over other antennas are aimed to present. For this purpose, a comparison is provided in Table 3, which includes as many performance characteristics as possible. Here, the performance values shown on the other rows of Table 3 are given for the frequency ranges of the antennas stated in the first row.

Table 3: Comparison of antennas in this study and the antennas in other relevant references.

\begin{tabular}{|c|c|c|c|c|c|}
\hline & $\begin{array}{l}\text { This } \\
\text { study }\end{array}$ & [14] & [15] & [16] & [17] \\
\hline $\begin{array}{c}\text { Band (10 } \\
\text { dB RL) } \\
{[\mathrm{GHz}]}\end{array}$ & $1.8-2.8$ & $2.5-2.7$ & $1.7-2.7$ & $1.7-2.2$ & $1.6-2.8$ \\
\hline $\begin{array}{l}\text { Minimum } \\
\text { Isolation } \\
{[\mathrm{dB}]}\end{array}$ & 20 & 40 & 25 & 30 & 35 \\
\hline $\begin{array}{c}\text { Peak } \\
\text { Gain } \\
\text { Range } \\
{[\mathrm{dBi}]}\end{array}$ & $2.9-6.8$ & $2-4.1$ & 8.1-8.9 & $2-5.6^{(1)}$ & $7.8-8.2$ \\
\hline $\begin{array}{c}\text { Minimum } \\
\text { XPD in } \\
120^{\circ} \\
\text { beam } \\
{[\mathrm{dB}]}\end{array}$ & 8.5 & $20^{(2)}$ & 12.7 & 14.7 & 10.2 \\
\hline $\begin{array}{c}\text { Total } \\
\text { Size } \\
{[\mathrm{cm} \times \mathrm{cm}]}\end{array}$ & $14 \times 14$ & $5.8 \times 5.8$ & $14 \times 14$ & $15 \times 15$ & $14 \times 14$ \\
\hline $\begin{array}{l}\text { Radiator } \\
\text { Size } \\
{[\mathrm{cm} \times \mathrm{cm}]}\end{array}$ & $5.7 \times 5.7$ & $4.5 \times 4.5$ & $14 \times 14$ & $5.7 \times 5.7$ & $14 \times 14$ \\
\hline
\end{tabular}

When the comparative results in Table 3 are examined, the proposed antenna has much wider bandwidth and higher peak gain values as compared to the studies of [14] and [16]. The isolation and XPD performances of [14] and [16] are observed to be better than the proposed antenna. However, the isolation and XPD values for [14] and [16] within the frequency band of $1.7-2.7 \mathrm{GHz}$ are probably much lower than those given in Table 3, and are expected to be close to these of the proposed antenna within 1.7-2.7 $\mathrm{GHz}$ frequency band. When the results of this study and the studies in [15] and [17] both having similar wide bandwidth of 1.7-2.7 GHz are compared, the antennas in [15] and [17] are superior to the proposed antenna in terms of isolation, gain and XPD values. However, these antennas have very severe drawback of having large radiator size. Their radiator sizes are about 2.5 times larger than the size of the proposed antenna, which brings more than $\lambda_{0} \times \lambda_{0}$ at the highest frequency of $2.7 \mathrm{GHz}$. Thus, by considering that the antenna arrays should be used along elevation (vertical) axis in base station antennas, this large size can cause significant grating lobes and multipath fading in elevation plane. The antennas in [15] and [17] might be appropriate when they are used as single antenna (without any array configuration) in the applications such as indoor mobile communication. Thus, as compared to the studies in [14-16], the proposed antenna can be concluded to have optimum performance in terms of frequency bandwidth, isolation, cross-polarization discrimination at azimuth plane and radiator size.

\section{Conclusions}

This study explains the design, production and results of a $\pm 45^{\circ}$ dual polarized antenna, which can be used in base station applications for GSM 1800/3G/LTE bands. The antenna consists of two printed and meandered dipoles perpendicular to each other above the ground plane to have a compact structure. In order to increase XPD value, which is a crucial parameter in dual polarized antennas, vertical parasitic elements acting as monopole are added. The realized antenna is measured to give more than $10 \mathrm{~dB}$ return loss and $20 \mathrm{~dB}$ isolation in the $1.8-2.8 \mathrm{GHz}$ band. Besides, the minimum XPD values are enhanced to $8 \mathrm{~dB}$ and $10 \mathrm{~dB}$ for the beamwidth of $120^{\circ}$ within the frequency bands of 1.7-2.7 GHz and 1.7-2.4 GHz, respectively where they are just $2 \mathrm{~dB}$ without parasitic elements. The gain values in the band are varied between $2.9 \mathrm{dBi}$ and $6.8 \mathrm{dBi}$.

As compared to similar studies, the proposed antenna optimally provides wide frequency bandwidth, compact radiator size, better gain values, and sufficient $\mathrm{XPD} /$ port isolation performances. As a future work, the performance of the proposed antenna is going to be investigated in the array (such as $2 \times 1$ array or $4 \times 1$ array) in order to be more appropriate for antennas used in base station applications.

\section{References}

[1] J. J. A. Lempiainen and J. K. Laiho-Steffens, "The performance of polarization diversity schemes at a base station in small/micro cells at $1800 \mathrm{MHz}$," IEEE Trans. Vech. Tech., vol. 47, no. 3, pp. 1087-1092, 1998. doi:10.1109/25.704863

[2] Z.-N. Chen and K.-M. Luk, Antennas for Base Stations in Wireless Communications, New York, USA: McGraw-Hill, 2009.

[3] M. Li and K. M. Luk, "Wideband magnetoelectric dipole antennas with dual polarization and circular polarization," IEEE Antennas Prop. Mag., vol. 57, no. 1, pp. 110-119, 2015. doi:10.1109/MAP.2015.2397091

[4] S. Zuo, Q. Q. Liu, and Z. Y. Zhang, "Wideband dualpolarized crossed-dipole antenna with parasitical crossed-strip for base station applications," Progress in Electromagnetics Research C, vol. 48, pp. 159-166, 2014. doi:10.2528/PIERC14021101 
[5] Y. H. Huang, Q. Wu, and Q. Z. Liu, "Broadband dualpolarised antenna with high isolation for wireless communication," IET Electron. Lett., vol. 45, no. 14, pp. 714-715, 2009. doi: 10.1049/el.2009.1256

[6] K. Ghorbani and R. B. Waterhouse, "Dual polarized wide-band aperture stacked patch antennas," IEEE Trans. Antennas Propag., vol. 52, no. 8, pp. 2171-2174, 2004. doi: 10.1109/TAP.2004.832484

[7] C. Sim, C. Chang, and J. Row, "Dual-feed dualpolarized patch antenna with low cross polarization and high isolation," IEEE Trans. on Antennas \& Propagation, vol. 57, no. 10, pp. 3321-3324, 2009. doi:10.1109/TAP.2009.2028702

[8] B. Li, Y. Z. Yin, Y. Zhao, Y. Ding, and R. Zou, "Dualpolarised patch antenna with low cross-polarisation and high isolation for WiMAX applications," IET Electronics Letters, vol. 47, no. 17, pp. 592-593, 2011. doi:10.1049/el.2011.1814

[9] T. İsenlik, M. M. Bilgiç, K. Yeğin, and M. Çiydem, "GSM/UMTS dual polarization base station antenna design," in General Assembly and Scientific Symposium XXXth URSI, Istanbul, Turkey, pp. 1-4, 2011.

[10]Z. D. Bao, Z. P. Nie, and X. Z. Zong, "A broadband dual-polarization antenna element for wireless communication base station," in Proc. IEEE AsiaPacific Conf. Antennas Propag., Singapore, pp. 144146, 2012.

[11] M. Kaboli, M. S. Abrishamian, S. A. Mirtaheri, and S. M. Aboutorab, "High-isolation XX-polar antenna," IEEE Trans. on Antennas \& Propag., vol. 60, no. 9, pp. 4046-4055, 2012. doi:10.1109/TAP.2012.2207059

[12] M. Secmen, and A. Hizal, "An inverted L-shape fed microstrip patch antenna for mobile communication," in IEEE 21st Annual International Symp. on Personal, Indoor and Mobile Radio Communications, Istanbul, Turkey, pp. 1102-1106, 2010.

[13]M. Secmen and A. Hizal, "A Dual-Polarized WideBand Patch Antenna for Indoor Mobile Communication Applications," Progress In Electromagnetics Res., vol. 100, pp. 189-200, 2010. doi:10.2528/PIER09112607

[14] T. Oh, Y. G. Lim, C.-B. Chae, and Y. Lee, "Dualpolarization slot antenna with high cross polarization discrimination for indoor small-cell MIMO system," IEEE Antennas and Wireless Propag. Lett., vol. 14, pp. 374-377, 2014. doi:10.1109/LAWP.2014.2364517

[15] Y. Luo, Q. X. Chu, and D. L. Wen, “A plus/minus 45 degree dual-polarized base-station antenna with enhanced cross-polarization discrimination via addition of four parasitic elements placed in a square contour," IEEE Trans. on Antennas and Propag., vol. 64, no. 4, pp. 1514-1519, 2016. doi:10.1109/TAP.2016.2522463

[16] Y. Luo, Q. X. Chu, and J. Bornemann, "Enhancing cross-polarisation discrimination or axial ratio beamwidth of diagonally dual or circularly polarised base station antennas by using vertical parasitic elements," IET Microwaves, Antennas and
Propagation, vol. 11 no. 9, pp. 1190-1196, 2017. doi:10.1049/iet-map.2016.0928

[17]Z. Tang, J. Liu, and Y. Yin, "Enhanced crosspolarization discrimination of wideband differentially fed dual-polarized antenna via a shorting loop," IEEE Antennas and Wireless Propag. Lett., vol. 17, no. 8, pp. 1454-1458, 2018. doi:10.1109/LAWP.2018.2849221

[18]C. A. Balanis, Antenna Theory Analysis and Design, $4^{\text {th }}$ edition, New Jersey, USA: Wiley, 2016.

[19]M. Secmen, "Multiband and Wideband Antennas for Mobile Communication Systems", in Recent Developments in Mobile Communications - $A$ Multidisciplinary Approach, Rejika, Croatia: Intech, 2011, pp. 158-159.

[20] J. Rashed and C. T. Tai, "A new class of resonant antennas," IEEE Transactions on Antennas and Propagation, vol. 39 no. 9, pp. 1428-1430, 1991. doi:10.1109/8.99054

[21] T. Endo, Y. Sunahara, S. Satoh, and T. Katagi, "Resonant frequency and radiation efficiency of meander line antennas," Electronics and Communications in Japan Part II: Electronics, vol. 83, no. 1, pp. 52-58, 2000. doi:10.1002/(SICI)15206432(200001)83:1<52::AID-ECJB7>3.0.CO;2-7

[22] Dreamcatcher, Malaysia, "ME 1300: Radiation Pattern Plotting System Setup," [Online]. Available: https://www.dreamcatcher.asia/cw/products/1300

[23] M. Secmen, "Ultrawideband inverse scattering method for resonance region target recognition: Application to small-scale airplane targets with measured data," in $9^{\text {th }}$ European Conference on Antennas and Propagation EUCAP, Lisbon, Portugal, pp. 1-5, 2015. 